\title{
Non-cardiac chest pain and benign palpitations in the cardiac clinic
}

\author{
Richard Mayou, Bridget Bryant, Colin Forfar, David Clark
}

\begin{abstract}
Objectives-To assess the characteristics of consecutive patients referred from general practice with the presenting disorder of chest pain or palpitations, and to determine the outcome at six months and three years.

Setting-A single consultant teaching hospital cardiac clinic receiving new referrals from a health district.

Design-94 consecutive referrals by general practitioners to a cardiac clinic with the presenting disorder of chest pain or palpitations were assessed at first attendance (research interview, cardiologists' ratings, systematic medical case note information), home interview six months later, and by a postal questionnaire at three years.
\end{abstract}

Outcome measures-Physical and psychological symptoms, limitation of activities, satisfaction with care, and use of health care resources.

Results-39 patients were given a cardiac diagnosis and 51 patients were not given a cardiac or other major physical diagnosis. The non-cardiac group was more likely to be young women, and to report other physical symptoms and previous psychiatric problems. The cardiac and non-cardiac groups reported progressive improvement in presenting symptoms and disability at the six months and three year follow up, but little change in mental state. Even so, three quarters of the noncardiac subjects described continuing limitation of activities, concern about the cause of their symptoms, and dissatisfaction with medical care.

Conclusions-A substantial proportion of the consecutive referrals continued to describe symptoms and disability throughout the three years after clinic attendance. Outcome was poor for those who had negative investigations and were reassured that they had no cardiac disorder or other serious physical finding. These results have implications for defining the role of psychological assessment and for the formulation of cost effective clinical measures to (a) minimise disability associated with cardiac disorder; and $(b)$ prevent and treat handicaps in those without major physical diagnoses.

(Br Heart f 1994;72:548-553)
Reports of chest pain and palpitations are the most common reasons for referral from a general practice to cardiac clinics. Patients not given a cardiac diagnosis are known by clinicians to be difficult to manage. In particular, there is consistent evidence that patients with chest pain who are found to have normal coronary angiograms have a poor outcome and continue to report persistent symptoms, disability, and continuing concern about heart disease. ${ }^{1}$ Less is known about the much larger number of patients with non-cardiac chest pain who are seen in cardiac clinics but do not undergo angiography, ${ }^{23}$ and especially about patients who are reassured that their palpitations are not medically significant. ${ }^{4}$ There is therefore a need for further research directed to improving the differential diagnosis of noncardiac disorders, the recognition of those who might benefit from extra treatment, and to the development and evaluation of treatments suitable for use with large numbers of patients in routine clinical settings.

This paper describes the assessment and management of a cohort of new patients referred to a district hospital cardiac clinic and reports their symptomatic and functional outcome at six months and three years. Findings for those with and without cardiac disorders are compared, and issues relating to patients thought not to have heart disease are discussed:

(a) What is the size of the clinical problem? What proportion of the patients is reassured by clinic assessment that their chest pain or palpitations are not medically serious? How many continue to suffer physical symptoms, disability, and distress?

(b) What are the clinical or other characteristics of non-cardiac patients compared with those diagnosed as having definite or probable heart disease?

(c) Can we identify either at initial clinic attendance, or at follow up, a subgroup of patients with poor prognosis who might benefit from extra intervention?

(d) What are the clinical implications of the provision of improved, but cost-effective, care for large numbers of patients?

\section{Methods}

Consecutive patients referred by general practitioners to a consultant cardiologist with presenting disorders of chest pain or palpitations were identified at their outpatient attendance. 
Table 1 Characteristics of patients with cardiac diagnoses (definite or probable) and those with no cardiac or other major physical diagnosis. Values are No (\%) unless stated otherwise

\begin{tabular}{|c|c|c|}
\hline & Non-cardiac patients $(n=51)$ & Cardiac patients $(n=39)$ \\
\hline Mean (range) age (years) & $43 \cdot 5(21-65)$ & $53.4(39.69)$ \\
\hline Male & $22(43)$ & $23(59)$ \\
\hline \multicolumn{3}{|l|}{ Previous hospital care for current episode } \\
\hline Outpatient & $10(20)$ & $2(5)$ \\
\hline Inpatient & $2(4)$ & $4(10)$ \\
\hline Emergency & $3(6)$ & $3(8)$ \\
\hline \multicolumn{3}{|l|}{ General practice care } \\
\hline Cardiac drugs & $20(39)$ & $22(56)$ \\
\hline Psychotropic drugs & $4(8)$ & $-(-)$ \\
\hline Diagnosis of heart disease & $6(12)$ & $11(28)$ \\
\hline \multicolumn{3}{|l|}{ Time before referral (months) } \\
\hline$<6$ & $24(47)$ & $17(44)$ \\
\hline $6-12$ & $8(16)$ & $7(20)$ \\
\hline$>12$ & $19(37)$ & $15(40)$ \\
\hline \multicolumn{3}{|l|}{ Symptoms } \\
\hline Mean (SD) Beck depression inventory & $4 \cdot 96(4 \cdot 33)$ & $4.59(4 \cdot 08)$ \\
\hline Mean (SD) Spielberger trait anxiety & $39 \cdot 00(11 \cdot 16)$ & $37 \cdot 10(10 \cdot 74)$ \\
\hline Mean (SD) hyperventilation score & $12.24(10.56)$ & $9 \cdot 72(7 \cdot 26)$ \\
\hline DSMIIIR psychiatric diagnosis & $22(42)$ & $9(24)$ \\
\hline \multicolumn{3}{|l|}{ Cardiologists' assessment and notes } \\
\hline Mental state (moderate/severe) & $20(39)$ & $7(18)$ \\
\hline Hyperventilation (possible/probable) & $13(26)$ & $4(10)$ \\
\hline Psychiatric contribution to symptoms (probable/definite) & $17(33)$ & $5(13)$ \\
\hline Current psychiatric problem & $22(42)$ & $6(16)$ \\
\hline Social problem & $11(21)$ & $4(11)$ \\
\hline \multicolumn{3}{|l|}{ Investigations } \\
\hline Exercise test & $25(49)$ & $26(67)$ \\
\hline 24 hour tape & $8(16)$ & $5(13)$ \\
\hline Angiography & $1(2)$ & $10(25)$ \\
\hline
\end{tabular}

Subjects were interviewed using a brief semistructured interview derived from previous work with angina ${ }^{5}$ and non-cardiac chest pain ${ }^{6}$ and completed three standard self report questionnaires: the Beck depression inventory, ${ }^{7}$ the Spielberger trait anxiety, ${ }^{8}$ and a hyperventilation check list. ${ }^{9}$ The cardiac assessment by a single consultant (CF) followed normal clinical practice but, in addition, the cardiologist completed a single page standard assessment sheet at the end of the consultation covering the physical diagnosis, aetiology of the present symptoms, hyperventilation, and role of psychological factors. Further information was abstracted from medical case notes. RM subsequently made psychiatric diagnoses according to the standard American diagnostic and statistical manual IIIR diagnostic criteria ${ }^{10}$ for psychiatric disorders.

Six months later subjects were reassessed at home. The self report questionnaires were repeated and a semistructured interview covered symptoms, beliefs, consultation, and disability. At three years subjects were sent postal questionnaires covering the same areas as the previous assessments, together with the Beck and Spielberger scales. Non-responders were contacted by telephone.

Statistical analysis was by the $\chi^{2}$ test and by generalised linear interactive modelling.

\section{Results}

Most patients had been managed in general practice for several months before referral (table 1) and were eventually referred to establish a definite diagnosis for persistent symptoms. Clinical diagnosis was usually established within two clinic visits. Thirty nine of the 94 (41\%) consecutive referrals were given either a definite or a probable cardiac diagnosis (cardiac group). Four further patients were given other specific physical diagnoses (two oesophagitis, two pneumonia).
Fifty one (54\%) were given no physical diagnosis (non-cardiac group). Most of those with cardiac diagnoses, but few of the non-cardiac patients, were offered continuing clinic follow up.

\section{CARDIAC GROUP}

The cardiologist diagnosed heart disease in 39 patients, $31(79 \%)$ with angina (24 definite and seven probable) and eight (21\%) with paroxysmal arrhythmias (six definite and two probable). Table 1 gives their characteristics. Physical symptoms were usually marked (figure): $17(43 \%)$ rated their symptoms as subjectively "severe", $11(28 \%)$ as "very distressing", and $8(21 \%)$ as "very limiting". The means of the self report anxiety and depression scores were within the upper normal range for the general population, ${ }^{78}$ and we made a research diagnosis of psychiatric disorder (four major depression and five anxiety disorders) in a quarter of patients. Reactions to the diagnosis of heart disease varied. Five patients found the definite diagnosis of heart disease a relief after months of uncertainty. Ten patients described considerable, but transient, distress. Two, both of whom had long histories of chronic psychological problems, reported continuing severe worry.

\section{Outcome}

Patients with cardiac disorders continued to use cardiological and general practice resources throughout the three year follow up period. Outcome was generally encouraging, but a small minority had disproportionate disability and appeared to pose management problems for other than strictly physical reasons.

At six months one patient had been rediagnosed as "non-cardiac" having had a normal coronary angiogram. Twenty nine patients $(74 \%)$ were still attending the clinic and 31 (79\%) were receiving regular cardiac drugs. 
Table 2 Outcome at six months for cardiac and non-cardiac patients. Values are No (\%) unless stated otherwise. Percentages are for all those interviewed or for total number of those for whom applicable

\begin{tabular}{llc}
\hline & $\begin{array}{l}\text { Non-cardiac patients } \\
(n=46)\end{array}$ & $\begin{array}{c}\text { Cardiac patients } \\
(n=29)\end{array}$ \\
\hline Symptoms & & \\
Improved (better/much better) & $27(59)$ & $14(48)$ \\
Symptoms in last month & $35(76)$ & $23(79)$ \\
Effects on social activities (moderate/great) & $11 / 44(26)$ & $17 / 28(61)$ \\
Walking two miles & $5 / 46(11)$ & $11 / 29(38)$ \\
Household tasks & $4 / 40(9)$ & $7 / 29(24)$ \\
Social life & $3 / 33(9)$ & $8 / 25(32)$ \\
Sex & $7 / 36(19)$ & $6 / 24(25)$ \\
Driving & $8 / 41(20)$ & $4 / 26(15)$ \\
Family life & $9 / 37(24)$ & $8 / 19(42)$ \\
Work & $4 \cdot 74(4 \cdot 61)$ & $5 \cdot 31(4 \cdot 41)$ \\
Mean (SD) Beck depression inventory & $38 \cdot 13(11 \cdot 62)$ & $37 \cdot 59(10 \cdot 39)$ \\
Mean (SD) Spielberger trait anxiety & $11 \cdot 0(9 \cdot 72)$ & $9 \cdot 97(8 \cdot 21)$ \\
Mean hyperventilation & & \\
Medical care & $25(54)$ & $7(24)$ \\
Dissatisfaction & $16(35)$ & $6(21)$ \\
General practitioner & $18(39)$ & $12(41)$ \\
Hospital & $21(46)$ & $13(45)$ \\
Reassured by clinic & $7(15)$ & $4(14)$ \\
Not reassured & $5 / 51(10)$ & $29 / 39(74)$ \\
Partially reassured & & \\
Very reassured & & \\
Attending clinic & &
\end{tabular}

Table 3 Outcome at three years for cardiac and non-cardiac patients. Values are No (\%) unless stated otherwise. Percentages are for all those interviewed or for total number of those for whom applicable

\begin{tabular}{lcc}
\hline & $\begin{array}{l}\text { Non-cardiac patients } \\
(n=40)\end{array}$ & $\begin{array}{l}\text { Cardiac patients } \\
(n=31)\end{array}$ \\
\hline $\begin{array}{l}\text { Symptoms last three months } \\
\text { Chest pain }\end{array}$ & $14(35)$ & $18(58)$ \\
Palpitations & $20(50)$ & $15(48)$ \\
Breathlessness & $17(43)$ & $16(52)$ \\
Mean (SD) Beck depression & $5 \cdot 33(4 \cdot 99)$ & $5 \cdot 06(4 \cdot 84)$ \\
Mean (SD) Spielberger anxiety & $39 \cdot 97(13 \cdot 70)$ & $38 \cdot 10(16 \cdot 54)$ \\
Medical care & $0(0)$ & $4(13)$ \\
Further angiography (last two years) & $10(25)$ & $5(16)$ \\
Would have liked more tests & $8(20)$ & $22(71)$ \\
Further cardiac treatment (last two years) & $0(0)$ & $9(29)$ \\
Currently attending oupatient clinic & $26(65)$ & $22(71)$ \\
Attended general practice in last three months & & 3 \\
Cardiac surgery & & 1 \\
Angioplasty & & 1 \\
Coronary artery bypass grafting & & 1 \\
ASD repair & & \\
Pacemaker & & \\
\hline
\end{tabular}

ASD, atrial septal defect.

Three patients had posed major non-medical management difficulties; one had refused further appointments, one was angry at being told that an operation was not required, and the third had multiple physical, psychological, and social difficulties.

Table 4 Non-cardiac group: comparison of those presenting primarily with chest pain and palpitations. Values are No(\%) unless stated otherwise

\begin{tabular}{lll}
\hline & Pain $(n=33)$ & Palpitations $(n=18)$ \\
\hline Mean (range) age (years) & $49 \cdot 6(22-69)$ & $41 \cdot 9(21-65)$ \\
Male & $20(61)$ & $2(11)$ \\
\hline Previous history & & \\
Symptom duration (months) & $15(46)$ & \\
$0-6$ & $6(18)$ & $9(50)$ \\
$7-12$ & $12(36)$ & $7(39)$ \\
>12 & $7(21)$ & $10(55)$ \\
Symptom characteristics & $19(58)$ & $13(72)$ \\
Severity (severe/very severe) & $13(39)$ & $5(28)$ \\
Distress (moderate/severe) & $7(21)$ & $6(33)$ \\
Limitation (moderate/severe) & $12(36)$ & $8(44)$ \\
Cardiologist's assessment & $10(30)$ & $9(50)$ \\
Hyperventilation (possible/definite) & $5(15)$ & $10(56)$ \\
Mental state (moderate/severe) & $12(36)$ & $10(55)$ \\
Psychiatric contribution (probable/definite) & $4 \cdot 89(4 \cdot 48)$ \\
Psychological assessment & $4 \cdot 79(4 \cdot 11)$ & $39 \cdot 67(11 \cdot 12)$ \\
Previous consultation for "nerves" & $13(38)$ & $9(50)$ \\
Psychological factors (general practitioner) & & \\
Mean (SD) Beck depression & & \\
Mean (SD) Spielberger anxiety & & \\
DSMIIIR diagnosis & & \\
\hline
\end{tabular}

The twenty nine subjects who were reinterviewed reported that their physical symptoms had improved (figure), although effects on everyday activities and social life remained marked (table 2). Most patients were satisfied with their medical care, but five reported side effects of $\beta$ blockers. There was little change in anxiety, depression, and hyperventilation scores.

At three years there had been no deaths and further overall clinical improvement (table 3). Four patients had had angiography since the six month assessment, and six had had an operation; one patient was awaiting coronary artery surgery. Seventy per cent were taking regular drugs for angina.

A fifth of the thirty one patients (78\%) who completed follow up questionnaires were symptom free and two fifths were not limited. Although most were satisfied with their progress and medical care, a fifth (six patients) described considerable distress and frustration at the effects of their symptoms on their everyday lives (figure).

\section{NON-CARDIAC GROUP}

Fifty one patients were given no major physical diagnosis. Although all were new referrals to the clinic, many had already received considerable medical care for their symptoms (table 1). Thirteen (25\%) had previously attended hospitals during the current episode, six $(12 \%)$ had been told by their general practitioners that they had heart disease, and 20 $(40 \%)$ were being treated with cardiac drugs. Five patients had had previous prolonged periods of chest pain or palpitations, during which four had definitely been told they did not have heart disease.

We rated the presenting symptoms as less limiting than those of the cardiac group (figure), but as no less subjectively severe or distressing. Overall the psychological and social characteristics were similar to those of the cardiac group, but the non-cardiac patients were more likely to be women $(p<0.05)$, young $(p<0.05)$, and have no family history of heart disease $(p<0.05)$. They were more likely to report multiple physical disorders and to be aware of heart disease in either relatives or other people. Although mean anxiety and depression scores were similar to those of the cardiac group, general practitioners and cardiologists recognised significantly $(p<0.05)$ more psychological problems among the noncardiac patients. Twenty one patients $(42 \%)$ were given research diagnoses of psychiatric disorders (anxiety and depression).

\section{Chest pain and palpitations}

These two subgroups were similar (table 4). The patients with "benign" palpitations were more likely than those with non-cardiac pain to be women $(p<0.05)$, younger $(p<0.05)$, and to report previous $(\mathrm{p}<0.05)$ psychiatric problems.

The cardiologist rated $20(60) \%$ of those with the primary disorder of chest pain as having minor physical explanations of their symptom: chest wall problems (11 patients); 
oesophageal reflux or spasm (three patients); cervical spine disorders (one patient); infections (two patients). They also often rated hyperventilation $(26 \%)$ and psychological factors $(33 \%)$ as probable contributing factors.

All 18 patients with the primary disorder of palpitations were rated by the cardiologist as being abnormally aware of cardiac rate or rhythm, six of sinus tachycardia, and 12 of ventricular ectopics. They rated psychological factors as important in seven (39\%). Thirteen (33\%) of those with chest pain were given research psychiatric diagnoses (nine major depression and four anxiety disorders). Nine $(50 \%)$ of those presenting with palpitations were given research psychiatric diagnoses (eight with panic disorder and one major depression).

\section{Outcome}

Outcome over the three year follow up was disappointing, with many patients reporting continuing symptoms, emotional stress, limitations of everyday activities, and concern about heart disease (tables 2 and 3). At 6 months only five $(10 \%)$ were still attending the clinic; four were still receiving cardiac drugs. The $46(90 \%)$ patients who were reinterviewed were no more likely than the cardiac patients to report improvement in their presenting symptoms (figure) or in their mood or hyperventilation scores, and the effects on everyday life remained considerable (table 2). Many patients were markedly dissatisfied with medical care in general practice $(n=25,54 \%)$ and hospital $(n=16,35 \%)$, particularly reporting that they had not been given adequate explanations for their continuing symptoms. They remained worried that the symptoms were due to cardiac or other serious physical causes. There were few differences between those who had presented with chest pain and those with palpitations, although the former reported more social disability.

At three years there had been no deaths and a further improvement in symptoms and functional capacity (table 3 ), but there was a considerable continuing use of hospital and general practice resources. Most of the 40 patients $(80 \%)$ who completed questionnaires reported persistent symptoms, although there had usually been an improvement in the severity of emotional distress and in everyday activities. Individual patterns of outcome were little changed from six months. All those who had a generally unsatisfactory outcome at three years had reported similar problems in the earlier follow up. Four patients remained convinced that heart disease was the cause of their continuing symptoms and a further eight believed it might be a cause. Other causes mentioned were: stress (18 probable, nine possible), indigestion (five), muscle pain (two), and infections (two). Ten patients said they did not know what had been the cause of their symptoms and several found continuing uncertainty unsatisfactory and worrying.

\section{Discussion}

We report a prospective and consecutive series of referrals from general practitioners to a single cardiologist providing a mainly district service. The findings have implications for the systematic development and evaluation of improved services. Our study has the advantage of reporting outcome for patients receiving usual care in a routine cardiac clinic, the only change from normal clinical procedure being the one page research assessment form on which the cardiologist recorded clinical diagnosis, possible aetiological factors, and the role of hyperventilation and psychological factors. The main disadvantages are the subjective nature of the cardiological assessment (and of the "reassurance" given) and the lack of precise diagnostic criteria or systematic investigation, and uncertainty about whether the findings are representative. We believe our findings are consistent with published data, typical of the hospital's four consultant clinics, and also accord with wider clinical experience, but they will need to be repeated and extended in further systematic multicentre research.

\section{CLINICAL IMPLICATIONS FOR THOSE WITH CARDIAC DIAGNOSES}

The clinical outcome of these patients was encouraging. Patients felt reassured, even relieved, to have been given a definite diagnosis and authoritative advice and treatment. Two potentially avoidable problems were apparent (a) lengthy waiting lists for angioplasty and on operation caused distress and resulted in serious work and other practical difficulties; (b) a minority either did not comply with medical advice or described "disproportionate" limitations of their everyday lives. Such difficulties might be prevented or reduced by early recognition and the provision of extra help (information, advice, exercise training, or psychological intervention) in the cardiac clinic, rehabilitation unit, or in primary care.

\section{SIZE OF THE PROBLEM OF NON-CARDIAC} DISORDERS

More than half of the new referrals presenting to a cardiac clinic with either chest pain or palpitations did not receive a major physical diagnosis. Despite reassurance, most of these patients described continuing symptoms, disability and distress six months and three years later. These findings for consecutive attenders are consistent with other evidence for more selected patients with non-cardiac chest pain ${ }^{111}$ and provide evidence of a similarly poor outcome for those with palpitations. The continuing management of the patients poses a considerable problem in primary care.

\section{CHARACTERISTICS OF PATIENTS WITH} NON-CARDIAC PAIN OR BENIGN PALPITATIONS We have previously argued that functional somatic symptoms, such as persistent noncardiac chest pain and benign palpitations, are of multicausal aetiology and that patients 
misinterpret minor physical pathology or the physiological consequences of autonomic arousal as evidence of heart disease. ${ }^{111}$ Misinterpretation is most likely in those who are predisposed by a hypochondriacal disposition, are worried about life stresses, have personal knowledge of heart disease in their own family or in other people, or have a psychiatric disorder. Patients fears are likely to be exacerbated and maintained by the delay in establishing a diagnosis. By the time they are given a specialist opinion, a patient may have been given a provisional diagnosis of heart disease in general practice and been prescribed cardiac drugs during a long wait for a specialist appointment and any subsequent investigation. Such delay and uncertainty inevitably increases anxiety (and its associated physical symptoms) and reinforces concern about heart disease.

It is apparent that the cardiologists implicitly used this model as minor physical factors, excessive awareness of cardiac rhythm, hyperventilation, and psychological factors were rated as aetiological factors in most patients. The application of this diagnostic formulation was clearly more straightforward in those with palpitations, where the association between anxiety and excessive awareness of rhythm is often clearer, than for chest pain where the role of physical factors over a period of months or even years is more complex and clinically more difficult to establish.

Despite the different aetiology, there were marked similarities between patients diagnosed as having heart disease and the noncardiac group. In particular, there was no systematic difference in mental state. This means it will not be possible to devise a simple psychological assessment which could identify patients with non-cardiac disorders. Greater concentration on the psychiatric history and psychological symptoms might enable more positive diagnoses of psychiatric disorders, such as depressive illness and panic disorder.

CAN NON-CARDIAC PATIENTS AT RISK OF POOR OUTCOME BE PREDICTED?

Our analyses show that it is difficult to predict symptomatic outcome at clinic attendance, apart from a small proportion of patients with long histories of multiple unexplained somatic symptoms, or who have not been reassured by previous negative investigations. We therefore believe that it is clinically more sensible and cost effective to concentrate on the early detection of persistent problems during follow up after clinic assessment. Patients who described difficulties at three months almost invariably described similar difficulties at three years, whereas those who were improved at three months continued to have an excellent outcome.

\section{WHAT ARE THE CLINICAL IMPLICATIONS FOR} NON-CARDIAC DISORDERS?

Negative clinical investigation and authoritative reassurance by a cardiologist is only effective for a minority of patients. The content of this reassurance and the way it is presented are likely to be crucial. Those who continue to experience symptoms say that they want an explanation which reassures them that they do not have a serious medical problem, and advice about how to return to a full life. We believe this can best be provided by: (a) simple measures feasible within the cardiac clinic together with reinforcement by the general practitioner; and $(b)$ the early recognition and selective more intensive treatment of persistent problems.

\section{Routine care}

Resources and time in cardiac clinics are limited, especially at a time of increasing referrals. Even so, small changes in clinical practice and the use of self help materials would be possible and valuable (handouts, cassettes, audiotapes). Information should include: (a) reassurance that the presenting symptoms are a common and familiar clinical problem, that specialist assessment is appropriate, and that outcome is excellent; $(b)$ an explanation of the alternative causes; and (c) advice about how to cope with any further symptoms and how gradually to resume normal full activities.

There may be a role for appropriately trained specialist nurses who would work with supervision from cardiologists and a behavioural psychologist. We are therefore currently evaluating an improved single intervention for patients with chest pain and negative coronary angiograms which is administered by a specially trained ward nurse. Our initial impression is that standardised and systematic discussion, handouts, cassettes, and follow up telephone calls are popular with patients and their families, and can be integrated into ward nursing routine.

\section{Selective extra help}

A small proportion of patients may benefit from specific treatments, such as referral to a gastroenterologist or antidepressant drugs. More often treatment will need to be directed to correcting patients' misunderstanding and misinterpretations of their symptoms and to promoting management of anxiety symptoms and a graded return to normal activities.

We have previously shown that a systematic psychological treatment can be effective with patients referred from general practice with persistent non-cardiac chest pain, ${ }^{6}$ and our current research has replicated these findings in a cardiac clinic. Such intensive treatment is only required by the most disabled patients, and briefer and simpler help could usually be provided by a general practitioner or specialist nurse.

This prospective study has shown the considerable size of the clinical problem in hospital and primary care of patients with chest pain and palpitation not due to heart disease. It is likely that simple improvements in care would be effective in most patients, but a proportion will require specialist help. Although it would be inappropriate to argue that cardiologists should be responsible for longer term management, they have a central role in 
establishing the diagnosis and initiating the treatment. There is a need for collaboration with primary care providers to ensure that treatment is continued. We believe that further research should be directed towards evaluating cost effective interventions which could be delivered in general practice or by appropriately trained experienced nurses. It will be essential to establish training programmes and ensure regular skilled supervision. Improved care is likely to lead to a better outcome and to a reduction in the use of primary care and hospital resources.

This study was supported by a grant from the Oxford University Research and Equipment Committee.

1 Chambers JB, Bass C. Chest pain and normal coronary anatomy: review of natural history and possible aetiologic factors. Prog Cardiovasc Dis 1990;33:161-84.
2 Mayou RA. Chest pain in the cardiac clinic. $f$ Psychosom Res 1973;17:353-7.

3 Channer KS, James MA, Papouchado M, Russell Rees J. Failure of a negative exercise test to reassure patients Failure of a negative exercise test to reassure
with chest pain. $Q \mathcal{F}$ Med 1987;240:315-22.

4 Barsky AJ. Palpitations, cardiac awareness, and panic disBarsky AJ. Palpitations, cardiac awar
order. Am f Med 1992;92:31-4.

5 Mayou RA, Bryant B. Quality of life after coronary artery surgery. Qf Med 1991;239:239-48.

6 Klimes I, Mayou RA, Pearce MJ, Coles L, Fagg JR. Psychological treatment for atypical non-cardiac chest pain: a controlled evaluation. Psychol Med 1990;20: 605-11.

7 Beck AT. Depression inventory. Philadelphia: Center for Cognitive Therapy, 1978.

8 Spielberger CD, Gorsuch R, Lushene R. State-trait anxiety inventory manual. Palo Alto: Consulting Psychologist Press, 1970 .

9 Clark DM, Hemsley DR. The effects of hyperventilation: individual variability and its relation to personality. $\mathcal{f}$ individual variability and its relation to
Behav Ther Exp Psychiatry 1982;13:41-7.

10 American Psychiatry Association. Diagnostic and statistical manual of mental disorder. 3rd ed. Washington DC: American Psychiatric Association, 1987.

11 Mayou RA. Invited review: atypical chest pain. $f$ Psychosom Res 1989;33:373-406. 